\section{Fracture of a self-expanding metal stent used to manage a post-vertical sleeve gastrectomy stenosis}

A vertical sleeve gastrectomy performed on a 33-year-old obese woman was complicated by severe stenosis of the middle of the gastric tube and a fistula at the site of the stenosis that was communicating with a perigastric abscess. Initially the patient was managed conservatively with placement of a nasoenteric tube and administration of antibiotics. This led to clinical improvement, but a Gastrografin study 2 weeks later showed persistent gastric stenosis despite closure of the fis-

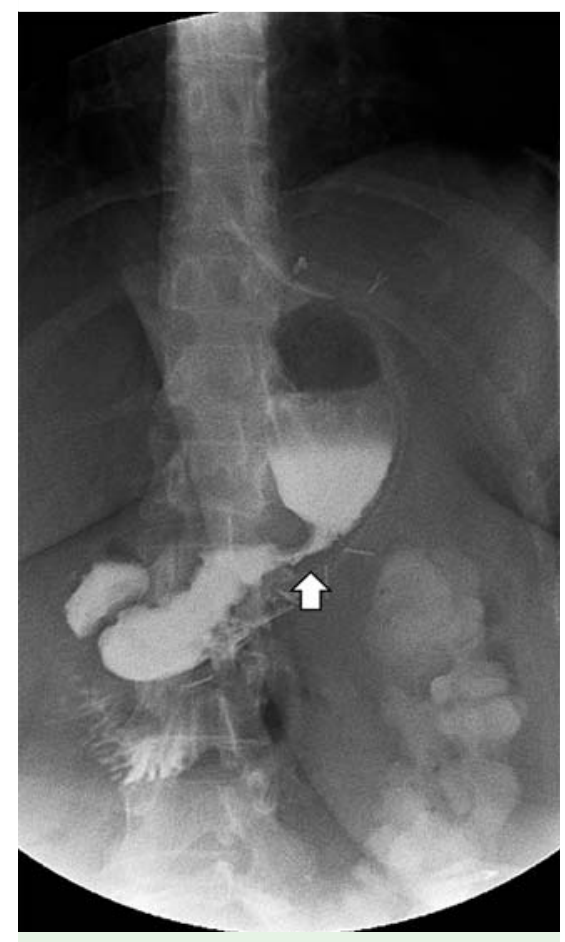

Fig. 1 Gastrografin contrast study showing a long stenotic segment in the middle third of the gastric tube.

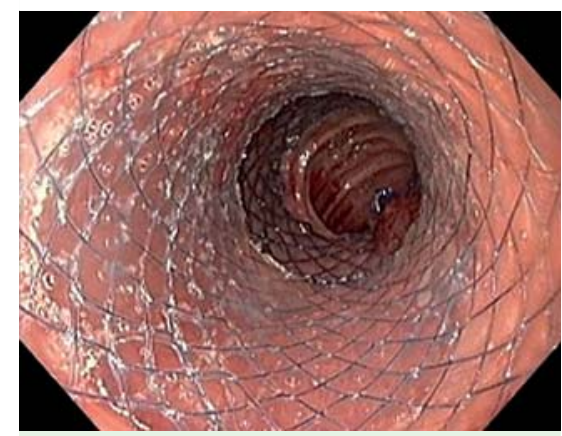

Fig. 2 Upper gastrointestinal endoscopy showing the distal portion of the opened self-expanding stent in the duodenum. tula ( $\bullet$ Fig. 1). An upper gastrointestinal endoscopy (UGE) confirmed a 5-cm stenosis of the gastric tube and a covered self-expanding metal stent (SEMS; Hanarostent Esophagus Benign BS; 22/28×120 $\mathrm{mm}$ ) was deployed across the stenosis (๑ Fig. 2).

The patient resumed oral intake after 48 hours and was discharged 5 days later. After 4 weeks, however, she developed abdominal pain and vomiting. A further Gastrografin contrast study suggested the

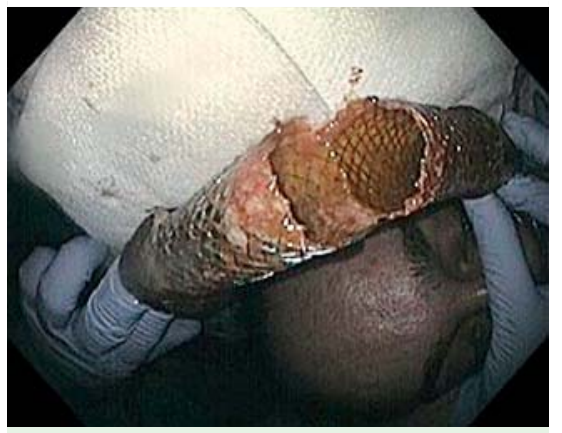

Fig. 4 The fractured self-expanding metal stent following its successful endoscopic removal.
SEMS had fractured ( $\bullet$ Fig. 3 ) and this was confirmed by computed tomography (CT) scanning. The SEMS was removed uneventfully during a further UGE ( $\bullet$ Fig.4). The gastric stenosis was adequately dilated and the patient was again discharged 3 days later.

Complications that can be attributed to SEMSs include obstruction, migration, bleeding, perforation, and stent fracture. Stent fracture can occur in patients with stenosis due to both malignant and benign etiologies. Potential causes of stent fracture include mechanical pressure, gastric acid-related corrosion, tumor ingrowth or overgrowth, mucosal hyperplasia, and food impaction.

\section{Endoscopy_UCTN_Code_CPL_1AH_2AJ}

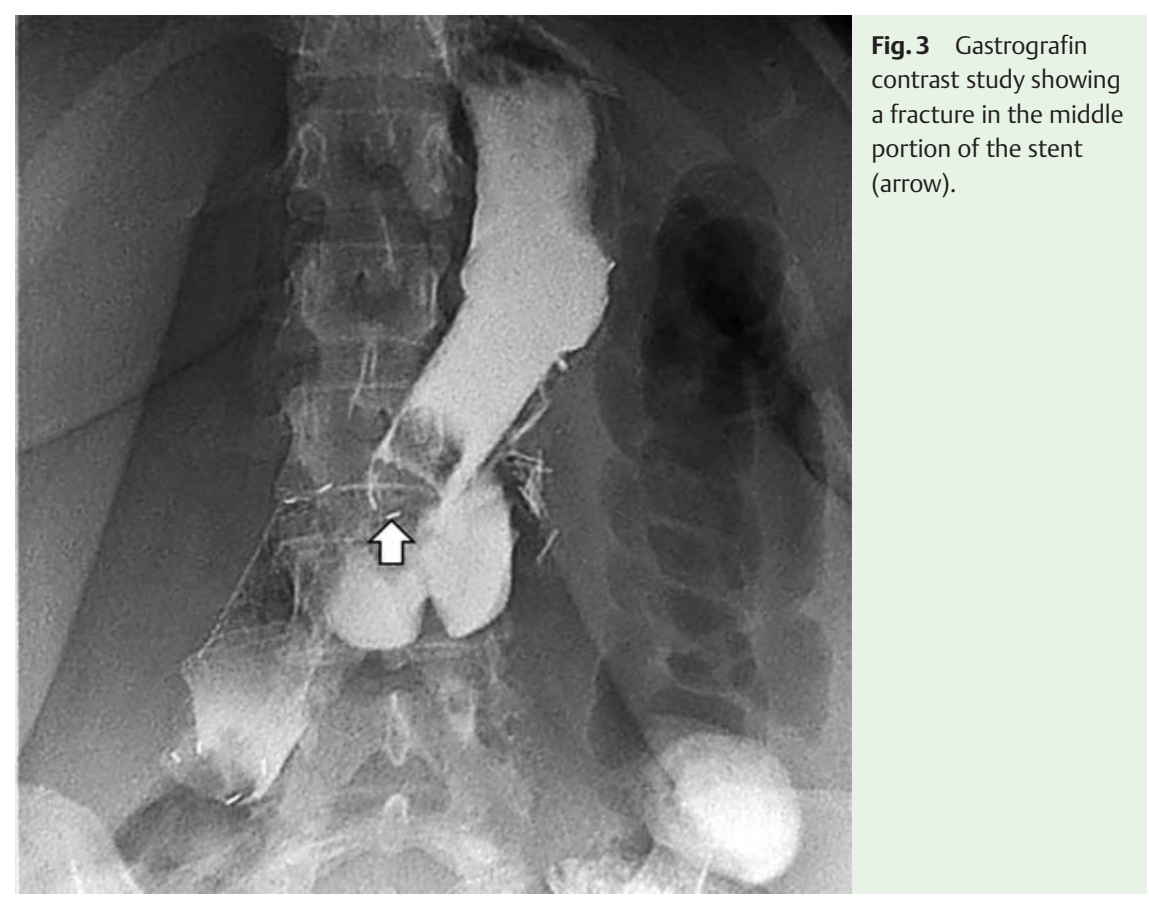


Competing interests: None

Samuel Fernandes ${ }^{1}$, Carlos Ferreira ${ }^{1}$, Joao Lopes ${ }^{1}$, João Raposo ${ }^{2}$, José Velosa ${ }^{1}$

${ }^{1}$ Department of Gastroenterology and Hepatology, Hospital Santa Maria, Lisbon, Portugal

2 Department of General Surgery, Hospital Santa Maria, Lisbon, Portugal
Bibliography

Dol http://dx.doi.org/

10.1055/s-0042-120265

Endoscopy 2016; 48: E372-E373

(c) Georg Thieme Verlag KG

Stuttgart · New York

ISSN 0013-726X
Corresponding author

Samuel Fernandes, MD

Hospital Santa Maria - Gastrenterologia e Hepatologia

Avenida Professor Egas Moniz Lisboa

Lisboa 1649-035

Portugal

Fax: +351-21-7805610

Samuelrmfernandes@gmail.com 